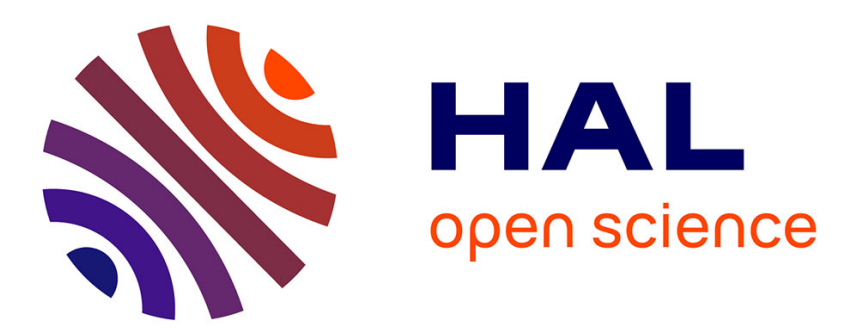

\title{
Influence of processing parameters on the macroscopic mechanical behavior of PVA hydrogels
}

\author{
Christopher Masri, Grégory Chagnon, Denis Favier
}

\section{To cite this version:}

Christopher Masri, Grégory Chagnon, Denis Favier. Influence of processing parameters on the macroscopic mechanical behavior of PVA hydrogels. Materials Science and Engineering: C, 2017, 75, pp.769776. 10.1016/j.msec.2017.02.045 . hal-01481365

\section{HAL Id: hal-01481365 https://hal.science/hal-01481365}

Submitted on 1 Apr 2018

HAL is a multi-disciplinary open access archive for the deposit and dissemination of scientific research documents, whether they are published or not. The documents may come from teaching and research institutions in France or abroad, or from public or private research centers.
L'archive ouverte pluridisciplinaire HAL, est destinée au dépôt et à la diffusion de documents scientifiques de niveau recherche, publiés ou non, émanant des établissements d'enseignement et de recherche français ou étrangers, des laboratoires publics ou privés. 


\title{
Influence of processing parameters on the macroscopic mechanical behavior of PVA hydrogels
}

\author{
Christopher Masri, Gregory Chagnon *, Denis Favier \\ ${ }^{a}$ Grenoble Alpes University, TIMC-IMAG, F-38000 Grenoble, France \\ ${ }^{\mathrm{b}}$ CNRS, TIMC-IMAG, F-38000 Grenoble, France
}

\begin{abstract}
This paper investigates the influence of three different processing parameters on the global mechanical behavior of PVA (Polyvinyl alcohol)/DMSO (Dimethylsulfoxide) hydrogels: the initial concentration of PVA, the DMSO: $\mathrm{H}_{2} \mathrm{O}$ ratio and the number of freeze/thaw cycle applied to the material. A specific thermo-regulated testing apparatus for hydrophilic materials is presented, along with the performed cyclic and rupture tests. The observed mechanical responses are explained by an in-depth analysis of the cross-linking phenomenon. Using the NeoHookean hyperelastic model, the experimental data is fitted and a link between the density of macromolecular chains in the material and its mechanical behavior is established. Strong differences are observed and discussed.
\end{abstract}

\section{Introduction}

Poly(vinyl alcohol) (PVA) hydrogels are used for various pharmaceutical [1] and biomedical [2] applications. It is a polymer of great interest since it is non-toxic, non-carcinogenic, bioadhesive and easy to process [3]. The elastic behavior of the hydrogel, its lifetime and the simplicity of its chemical composition result in its acceptance by the human body. Its stiffness, which can easily be controlled by adjusting its chemical composition, can compare well to the one of soft tissues. PVA is used in drug delivery systems [4], contact lenses [5], and substitutes tendons [6], ligaments [7], cartilage [8], and more.

PVA hydrogels can be prepared by various methods which impact their mechanical and optical properties. They can be cross-linked through the use of cross-linking agents, resulting in residual amounts of potentially toxic agents in the PVA gel [9]. Another method of chemical cross-linking includes the use of electron beam or $\gamma$-irradiation [10]. These methods have the advantage of not leaving behind potentially toxic agents but often result in bubble formation [10]. A "physical" cross-linking of the hydrogel is also possible [11] due to crystallite formation upon repeated freezing and thawing cycles. This method results in a high degree of swelling in water and an elastic nature. In addition, the properties of the hydrogel are influenced by the molecular weight of the polymer, the concentration of the aqueous PVA solution, the temperature and time of freezing and thawing and by the number of freezing/thawing cycles [12]. An improvement of mechanical properties

\footnotetext{
* Corresponding author at: TIMC-IMAG laboratory, Domaine de la Merci, 38706 La Tronche Cedex, France.

E-mail address: gregory.chagnon@univ-grenoble-alpes.fr (G. Chagnon).
}

when using a gelation process using an aqueous solution of dimethylsulfoxide (DMSO) has been reported [13]. This study aims at quantifying the effect of the processing parameters of this last process on the mechanical behavior of the ensuing hydrogel. To this day, the available information is limited to the evolution of the mechanical properties with aging [14], the effect of processing parameters on the structure [12] and basic tensile testing [13].

The correlation between the molecular weight and the size of crystallites in PVA polymers has already been established [15]. It is also known that there is a minimum PVA chain length necessary for crystallization [3]. For these reasons, it was chosen to study a PVA of specific molecular weight instead of evaluating the impact of the polydispersity of the polymer. This paper aims at studying the influence of the processing parameters on the mechanical behavior of specific PVA hydrogels. Section 2 presents the materials and the experimental setup. Section 3 gives the results obtained for different processing parameters. The evolution of the mechanical behavior is discussed in Section 4. Finally, concluding remarks close the paper.

\section{Experimental setup}

This section focuses on the presentation of the elaboration parameters of the hydrogel, its preparation, the experimental setup of this study and the description of the mechanical tests performed.

\subsection{Preparation of PVA hydrogels}

The polymer used in this study is a PVA (Mw 85,000-124,000, $99+\%$ hydrolyzed) from Sigma Aldrich. It was stored in a dry 
environment to prevent moisture absorption. DMSO (ACS reagent, $\geq$ 99.9\%) was also obtained from Sigma Aldrich. Solutions were prepared by heating a mixture of PVA in an aqueous medium of ultra-pure water and DMSO for $2 \mathrm{~h}$ at $120^{\circ} \mathrm{C}$ on a magnetic stirrer. DMSO is used to regulate the freezing of the aqueous medium, which would otherwise lead to phase separation and prevent the large formation of PVA crystallines [13]. The beaker used was covered to avoid loss of water and DMSO vapors. After the heating step, the PVA solutions were placed in a vacuum bell at room temperature to facilitate the release of air bubbles. The solutions were then injected into $2 \mathrm{~mm}$ thick rectangular molds and placed in a cooling oven in which freeze/thaw $\left(10 \mathrm{~h}\right.$ at $-20^{\circ} \mathrm{C}$ followed by $2 \mathrm{~h}$ at $20^{\circ} \mathrm{C}$ at a constant rate) cycles were achieved. The solutions' dosages and the number of applied cycles are listed in Table 1 . Since a physical cross-linking process is used to synthesize the polymers, no covalent bonds are formed between the hydrogel and the DMSO. The latter can thus be evacuated by repeated rinsing. The $2 \mathrm{~mm}$ rectangular hydrogel sheets were rinsed by exposing them to a flow of water for 5 min, 3 times per day the first week following synthesis. The polymers where then stored for 4 months in containers filled with distilled water, which was renewed every day. This ensured the extraction of any residual DMSO [12,13] and limited aging effects [14].

\subsection{Mechanical testing of the hydrogel}

Rectangular samples of $40 \mathrm{~mm}$ width and $10 \mathrm{~mm}$ height were then cut from the rinsed hydrogel sheets for pure shear testing and inserted in the sample holders. The resulting area of interest (AOI) is a $40 \mathrm{~mm}$ width and $2 \mathrm{~mm}$ height rectangle, as shown in Fig. 1.

Fig. 2 shows the tested sample in both its initial and deformed states. It is supposed that the sample stays rectangular and border effects are neglected [16]. The material deformation gradient tensor $\overline{\bar{F}}$ describes the motion of a continuum. It characterizes the transformation of a material point from the reference configuration to a deformed one. The material is considered incompressible, which translates into $\operatorname{det}(F)=1$. This implies that $\overline{\bar{F}}$ can be written as:

$F=\left[\begin{array}{ccc}\lambda & 0 & 0 \\ 0 & 1 & 0 \\ 0 & 0 & \lambda^{-1}\end{array}\right]$

where $\lambda=\frac{l}{l_{0}}$ is the stretch ( $l$ and $l_{0}$ are the initial and instantaneous height of the specimen, as presented in Fig. 2). The tests are analyzed by measuring the nominal stress defined as:

$\pi=\frac{F}{A_{0}}$

where $F$ is the applied force and $A_{0}$ the initial cross section.

Being hydrophilic, PVA hydrogels tend to dry up when removed from water. To attend this problem, a thermostatic tank was designed to conduct immersed mechanical tests at a controlled temperature on a Gabo Eplexor $500 \mathrm{~N}$ mechanical test machine fitted with a $10 \mathrm{~N}$ force transducer. Fig. 3 shows the device that consists of a hermetic steel tank, heat cartridges, a thermocouple sensor and a proportionalintegral-derivative closed loop controller to adjust the temperature of the tank. The tests were performed at a constant temperature of $37 \pm$ $1{ }^{\circ} \mathrm{C}$.

In order to investigate the behavior of the material, different types of mechanical tests are performed. The first consists in a series of 5 loadunload cycles reaching a stretch of $\lambda=1.25$ to analyze the cyclic behavior of the material. The second type is a load until failure in order to obtain the ultimate strain and stress of the material.

\section{Test results}

\subsection{Cyclic tests}

Results obtained on gels processed with a PVA concentration of $10 \mathrm{wt} \%$ and $5 \mathrm{wt} \%$ are presented in Figs. 4 and 5 respectively. The general shape of the curves is the same for all the tested materials, although the reached values differ. Hence the description of a single curve can apply to all the others. Focusing on $A_{1}$ for example, all the loading phases are superimposed except for the first one. This softening phenomenon is often present in polymeric material and is known as stress-softening [17]. In the present case, however, the phenomenon is small and can be neglected. The unloading phases are all overlaid. Also, the difference between the loading and unloading phases, however small it may be, can be associated to hysteresis which is characteristic of the behavior of viscoelastic materials. It can be noted that the hysteresis is very small. The viscoelastic phenomenon is negligible in comparison to the hyperelastic behavior. Nevertheless, a residual strain appears. The evolution of the phenomena mentioned earlier - i.e. stress level reached, hysteresis, residual strain and softening- will be evaluated function of the processing parameters.

Fig. 4a shows the mechanical response of materials composed of $10 \mathrm{wt} \%$ PVA and 80\% DMSO. The stress level reached for a stretch of 1.25 decreases with the number of freeze/thaw cycles. Residual strain and hysteresis, however, are maximal for 2 freeze/thaw cycles and minimal for 1 . Samples $A_{2}$ and $A_{3}$, unlike $A_{1}$, exhibit some stress softening.

The results for a DMSO concentration of $60 \%$ are presented in Fig. $4 \mathrm{~b}$. In this case the highest and lowest levels of stress are observed for 2 and 1 freeze/thaw cycles respectively. Residual strains behave similarly for materials containing $10 \mathrm{wt} \%$ PVA and 80\% DMSO, even though the hysteresis is slightly more important. Stress-softening, however, does not appear as the first load is confounded with the others.

In general, no significant trend can be extracted concerning the stress level reached achieved by materials containing 10 wt\% PVA. Hysteresis and residual strain, however, increase with the number of freeze/ thaw cycles. Stress-softening is only observed for $80 \%$ DMSO.

Fig. 5 presents the results of the pure shear cyclic tests on hydrogels containing $5 \mathrm{wt} \%$ PVA. Since materials $B_{1}$ and $E_{1}$ are so brittle, inserting them in the sample holders was problematic and a couple of loading cycles were enough to tear them. This explains why no cyclic data is provided for these materials.

For a DMSO concentration of $80 \%$ (Fig. 5a), the highest stress is achieved for 2 freeze/thaw cycles, but the variation is very small. Also both samples have the same residual strain, which can reach $10 \%$, and a very similar softening rate. The hysteresis is relatively large for the first load/unload cycle but becomes quasi-null for the following ones.

Table 1

Processing parameters of the PVA hydrogels.

\begin{tabular}{|c|c|c|c|}
\hline Sample & Initial PVA concentration in solution [wt\%] & Initial DMSO: $\mathrm{H}_{2} \mathrm{O}$ ratio & Number of freeze/thaw cycles \\
\hline$A_{1}, A_{2}, A_{3}$ & 10 & $80: 20$ & $1,2,3$ \\
\hline$B_{1}, B_{2}, B_{3}$ & 5 & $60: 40$ & $1,2,3$ \\
\hline$C_{1}, C_{2}, C_{3}$ & 10 & $80: 20$ & $1,2,3$ \\
\hline$D_{1}, D_{2}, D_{3}$ & 5 & $60: 40$ & $1,2,3$ \\
\hline$E_{1}, E_{2}, E_{3}$ & 5 & $40: 60$ & $1,2,3$ \\
\hline
\end{tabular}




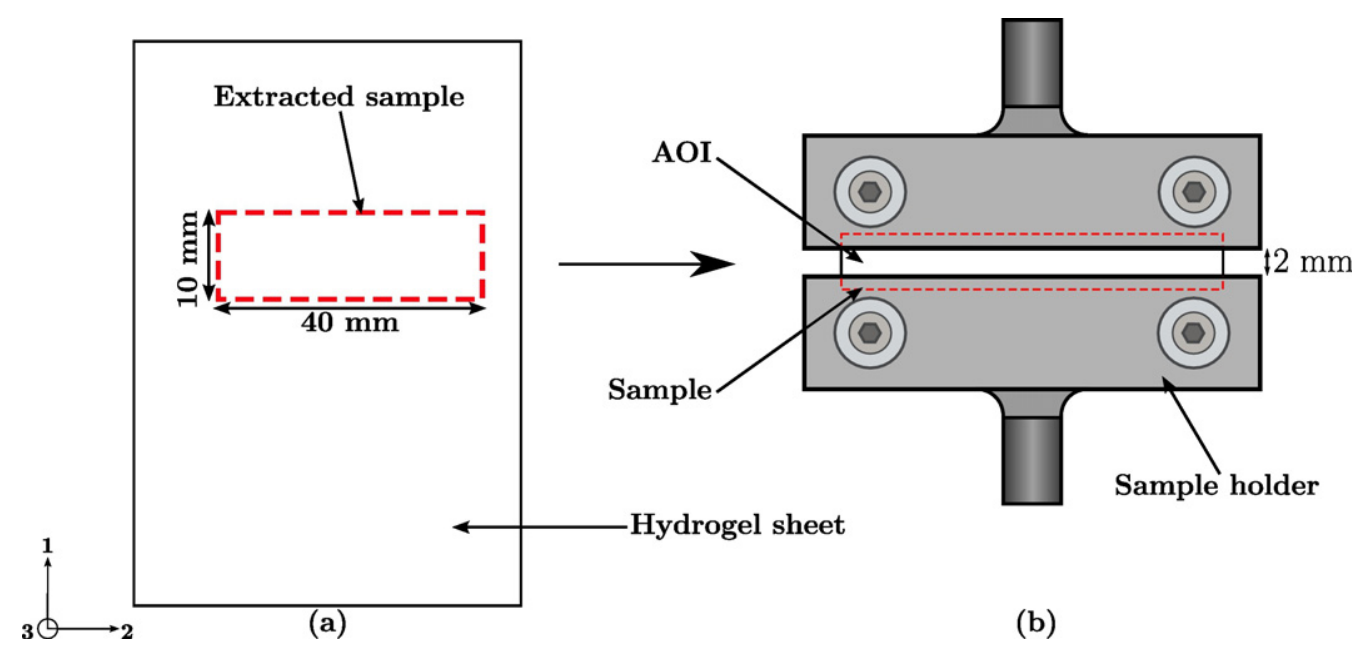

Fig. 1. Sample preparation: (a) extraction of the sample from the hydrogel sheet, (b) insertion of the extracted sample in the sample holders.

Fig. 5b shows that the highest stress level is reached by materials containing 60\% DMSO and increases with the number of freeze/thaw cycles, although in this case also, the variation is not very important. The residual strain, however, is maximal for sample $D_{3}$ and minimal for sample $D_{1}$. Softening increases with freeze/thaw cycles while hysteresis remains constant.

For material configuration $E$ (Fig. 5c), the highest stress level is reached by sample $E_{3}$. In comparison with material $E_{2}$, only a slightly more important softening can be observed while the residual strain is equivalent. Hysteresis however, does not manifest for a DMSO concentration of $5 \%$.

In general, for materials containing $5 \mathrm{wt} \% \mathrm{PVA}$, highest stresses are achieved for a DMSO concentration of $60 \%$ while the residual strain is maximal for $80 \%$ DMSO. These two statements compare well with the observations made on materials containing $10 \mathrm{wt} \%$ PVA. Softening can be observed for all material configurations except $D_{1}$, and hysteresis can almost be neglected.

To sum up, the initial PVA concentration has a consequent influence on the yielded stress by the material: on average, for a given DMSO: $\mathrm{H}_{2} \mathrm{O}$ ratio and a given number of freeze/thaw cycles, the maximum achieved stress is 3 times higher for a PVA concentration of $10 \mathrm{wt} \%$. The effect of DMSO seems maximal for a concentration of $60 \%$. The effect of the number of freeze/thaw cycles depends on the other two parameters.

\subsection{Ultimate values}

The analysis of the evolution of the ultimate stress and strain of the material gives a general overview of the effect of the processing parameters. Tables 2 and 3 show the results of the tests. Materials containing 10 wt\% of PVA, i.e. A and C compositions, reach higher ultimate strains and stress than gels containing $5 \mathrm{wt} \%$ of PVA.

Table 2 presents the results for hydrogels containing $10 \mathrm{wt} \%$ PVA. Its analysis shows that the ultimate stain seems to be increasing with the number of freeze/thaw cycles in an almost linear manner for materials containing $80 \%$ DMSO. The ultimate stress however, is maximal for 2 freeze/thaw cycles and quasi-equal for 1 and 3 cycles.

For hydrogels containing 60\% DMSO, the ultimate strain is maximal for $C_{1}$ and minimal for $C_{2}$, even though the difference in their values is relatively small, i.e. $21 \%$. The ultimate stress however, is maximal for 2 freeze/thaw cycles and minimal for 1 , albeit the difference between $C_{1}$ and $C_{3}$ being of $18 \mathrm{kPa}$ only.

Table 3 shows that the ultimate stress and strain increase with the number of freeze/thaw cycles for all three compositions. In this case also, materials containing 60\% DMSO achieve the highest ultimate stress for a given amount of freeze/thaw cycles, except for material $D_{1}$ which is slightly lower than $B_{1}$. Hydrogels containing $80 \%$ DMSO having undergone 1 or 2 freeze/thaw cycles reach the highest strains before breaking,

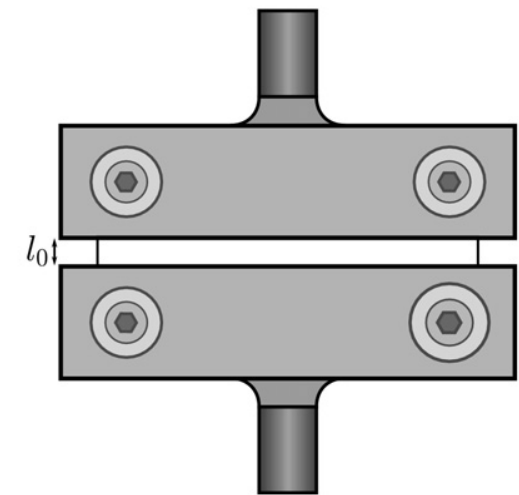

$\stackrel{1}{\stackrel{1}{\longrightarrow} 2}$ (a)

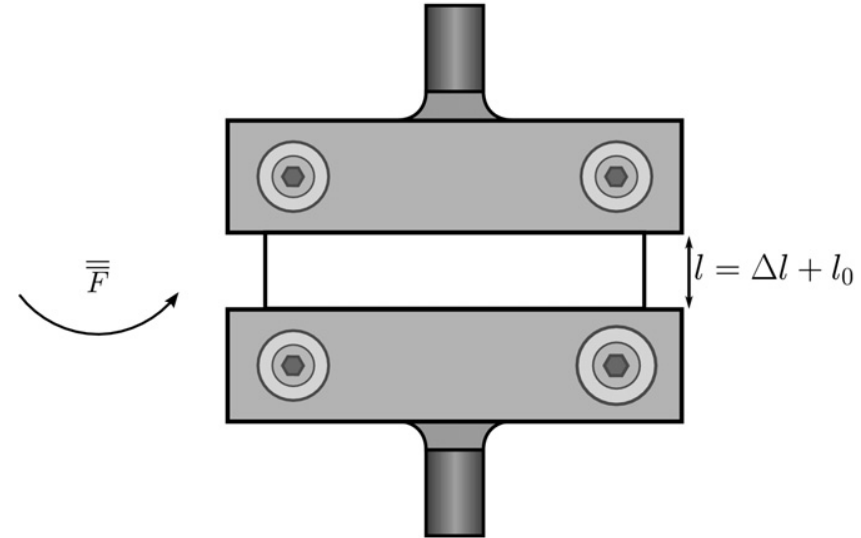

(b)

Fig. 2. Pure shear test: (a) initial configuration of the material, (b) deformed configuration of the material. 


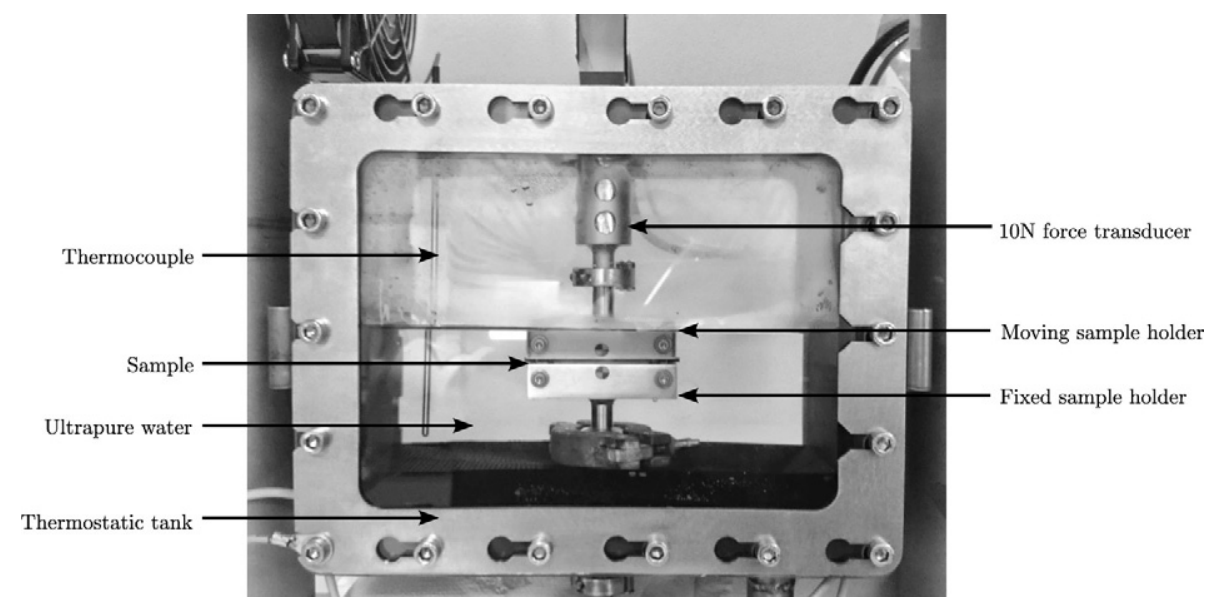

Fig. 3. The mechanical testing apparatus: the sample and its holders are inserted in a stainless steel tank containing a thermostatic aqueous medium.

while $D_{3}$, which contains $60 \%$ DMSO, achieves the highest ultimate strain for materials made with 3 cycles.

Fig. 6 shows a scatter of hydrogel compositions based on ultimate strain and stress. Higher PVA concentration clearly improves mechanical behavior. On average, a hydrogel containing a concentration of PVA of $10 \mathrm{wt} \%$ can yield ultimate stresses four times larger than hydrogels containing $5 \mathrm{wt} \%$. The number of freeze/thaw cycles, however, seems to improve the ultimate strain achieved by materials. A DMSO concentration of $60 \%$ produces materials that yield the highest stress for a given PVA dosage while ultimate strain seems to improve with freeze/ thaw cycles.

In summary, cyclic tests and failure tests lead to the same conclusion. Higher PVA concentration improves the mechanical behavior of the hydrogel. This trend was already observed by [12], even though the ultimate stresses for different materials measured in this study are significantly lower. The ultimate strains, however, are consistent.

The DMSO's influence varies with the quantity of PVA in the initial solution, but hydrogels containing 60\% DMSO yield the highest stresses.

The influence of the number of freeze/thaw cycles is difficult to assess for the cyclic tests as it did not seem to have much effect on hydrogels containing 5\% PVA and no clear trend could be extracted for materials made of $10 \%$ PVA.

\section{Discussion}

In this section, the cross-linking mechanism is first described in order to evaluate its impact on the mechanical behavior of the hydrogels when submitted to different tests. An explanation of the observed results is then proposed accordingly. Finally, the impact of the processing parameters on the mechanical properties of the hydrogel will be quantified by considering the Neo-Hookean model.

\subsection{Description of the cross-linking mechanism}

According to Yokoyama et al. [18], the hydrogel is composed of three kind of phases: the solution phases with a very low concentration of PVA, which corresponds to the crystal phases of ice in the frozen gel; the amorphous phases, in which every PVA chain is associated with water; and the PVA crystal phases, which restrain gross mobility of the amorphous chains.

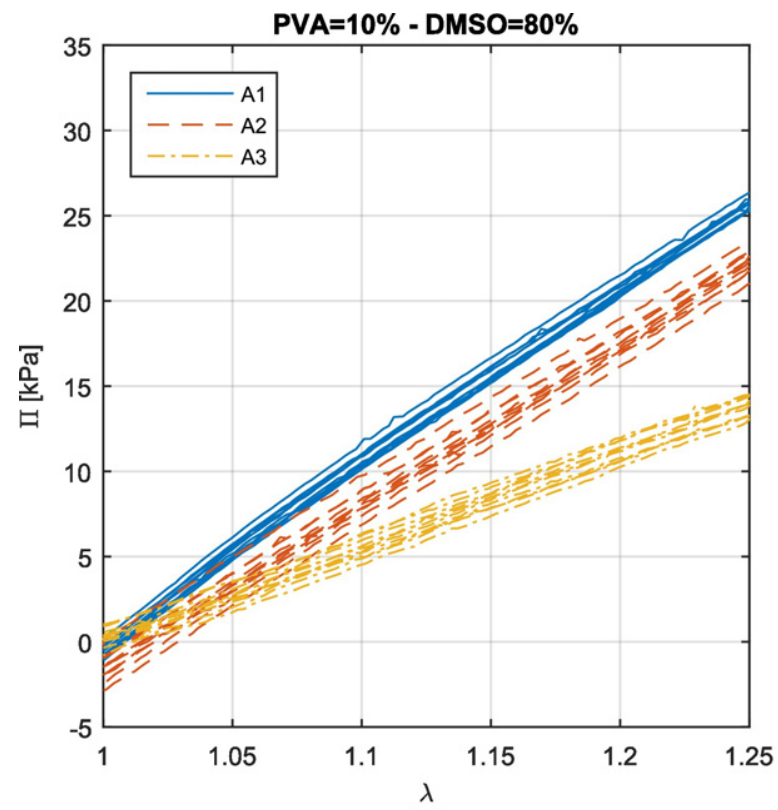

(a)

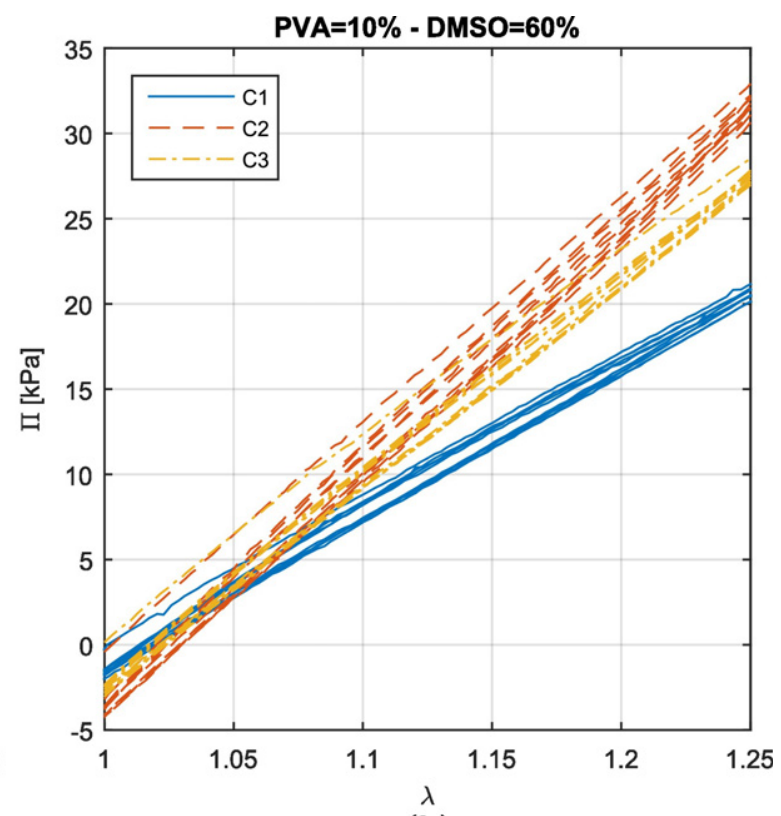

(b)

Fig. 4. Cyclic pure shear test results for a concentration of 10\% PVA and (a) 80\% DMSO and (b) 60\% DMSO. 


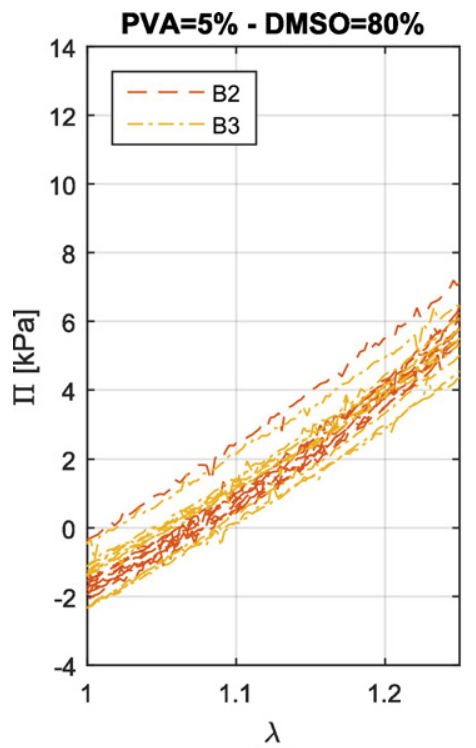

(a)

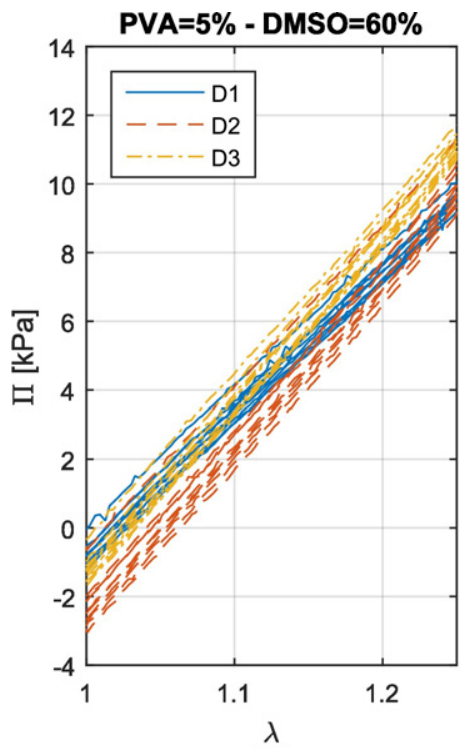

(b)

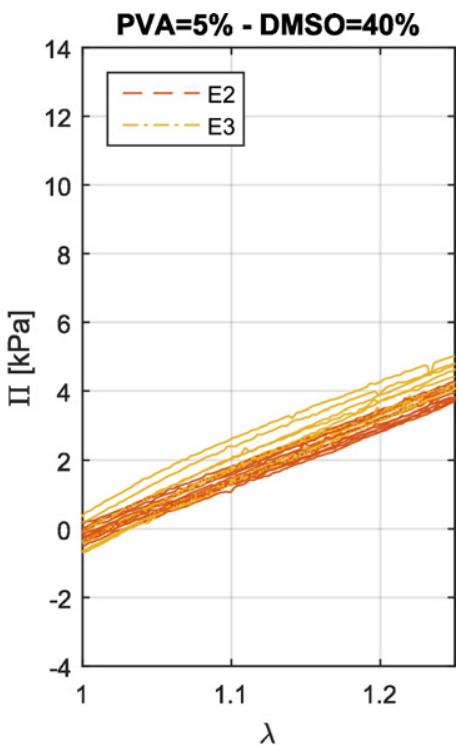

(c)

Fig. 5. Cyclic pure shear test results for a concentration of 5\% PVA and (a) 80\% DMSO, (b) 60\% DMSO and (c) $40 \%$ DMSO.

During the freezing process, the ice crystals formed by the solution phases force the amorphous chains - which are initially free and distributed homogeneously in the solution (water and DMSO in this study) - to become constricted in whatever space is not crystallized. The amorphous chains, associated with water through hydrogen bonds, maintain a fraction of the PVA in its amorphous state, which prevents complete crystallization of the polymer thus giving it a rubbery texture. The crystal phase consists of a high concentration of polymer chains linked to each other and excluding any trace of solution. By restraining the mobility of certain points of the structure, it ensures cross-linking. Repeating the freeze/thaw process should push more and more amorphous chains to the previously established amorphous phase, thus increasing its molecular concentration, while also increasing the size of the crystallines.

DMSO is used to prevent the freezing of the PVA solution by bonding to water molecules, thus preserving the amorphous phase and achieving cross-linking faster. Hoshino et al. [19] showed that at $23^{\circ} \mathrm{C}$, approximately 800,50 and 1100 min were needed to form gels containing $40 \%$, $60 \%$ and $80 \%$ DMSO respectively and $5 \mathrm{wt} \%$ PVA. Although cross-linked differently - at room temperature vs. $-20{ }^{\circ} \mathrm{C}$ - this might explain the fact that hydrogels containing 60\% DMSO exhibit better mechanical properties than others: the amorphous phase starts forming quicker which leads to denser polymer chains and crystallines.

The cross-linking mechanism is thus responsible for the cyclic response of the hydrogels: the amorphous chains being randomly organized, the first tensile load applied to the material is expected to unfold them to a certain measure. After unloading, the chains recover their initial state through elasticity, but are unable to retrieve their tangled configuration. Due to this new configuration of the amorphous phase, a smaller effort is needed to reach the desired strain thus explaining the difference between the first load and all the following loads. This explanation also accounts for the residual strain observed on the tested materials. The hysteresis, however small in the loading

Table 2

Ultimate stress and strain of the material for different processing parameters at a fixed PVA concentration of $10 \%$.

\begin{tabular}{lllllll}
\hline Sample ID & $A_{1}$ & $A_{2}$ & $A_{3}$ & $C_{1}$ & $C_{2}$ & $C_{3}$ \\
\hline Ultimate strain [\%] & 222 & 310 & 434 & 358 & 337 & 349 \\
Ultimate stress [kPa] & 188 & 230 & 189 & 198 & 292 & 216
\end{tabular}

range, is a result of the viscous behavior of the polymer chains of the amorphous phase. Stress softening might be caused by the rupture of some polymer chains during loading. Being fragile, the crystal phase might have also been damaged during cyclic loading, thus explaining the failures of materials $B_{1}$ and $E_{1}$.

For hydrogels containing $5 \mathrm{wt} \% \mathrm{PVA}$, the results concur with the observations of Hoshino et al. [19], i.e. the stress level reached is highest for a DMSO concentration of $60 \%$ and lowest for one of $80 \%$. The effect of freeze/thaw cycles is less obvious at this stretch level, but the fact that materials $B_{1}$ and $E_{1}$ did not achieve the cyclic test while materials $B_{2,3}$ and $E_{2,3}$ did, suggests that the resistance to tearing during cyclic loading is improved.

For hydrogels containing $10 \mathrm{wt} \%$ PVA also, the highest stress levels are also most important for the configuration containing 60\% DMSO except for material made by a single freeze/thaw cycle.

The response of the material to rupture tests can be explained by the fact that a larger PVA concentration ultimately leads to denser amorphous and crystal phases. More amorphous polymer chains increase the resistance to strain and lead to a larger yielded stress. This is easily visible on Fig. 6: on average, configurations $A$ and $C$ yield ultimate stresses four times larger than materials $B, D$ and $E$ and ultimate strains twice as big. For configurations $A, B, D$ and $E$, the ultimate strain achieved by most tested hydrogels improves with the number of freeze/thaw cycles. This can be explained by the increase of PVA chains in the crystal phase with the number of freeze/thaw cycles, resulting in an improvement of resistance to rupture of the hydrogels. For configuration $C$, achieved ultimate strains remain constant regardless of the number of freeze/thaw cycles. This might be due to a fully crosslinked structure after a single cycle. The variation of ultimate stress increases with the number of cycles for materials containing $5 \mathrm{wt} \%$ PVA. For hydrogel compositions $A$ and $C$, however, no clear trend can be extracted.
Table 3

Ultimate stress and strain of the material for different processing parameters at a fixed PVA concentration of $5 \%$.

\begin{tabular}{llllllllll}
\hline Sample ID & $E_{1}$ & $E_{2}$ & $E_{3}$ & $D_{1}$ & $D_{2}$ & $D_{3}$ & $E_{1}$ & $E_{2}$ & $E_{3}$ \\
\hline Ultimate strain [\%] & 154 & 218 & 261 & 84 & 187 & 323 & 72 & 117 & 200 \\
Ultimate stress [kPa] & 38 & 48 & 77 & 31 & 81 & 95 & 10 & 31 & 56 \\
\hline
\end{tabular}




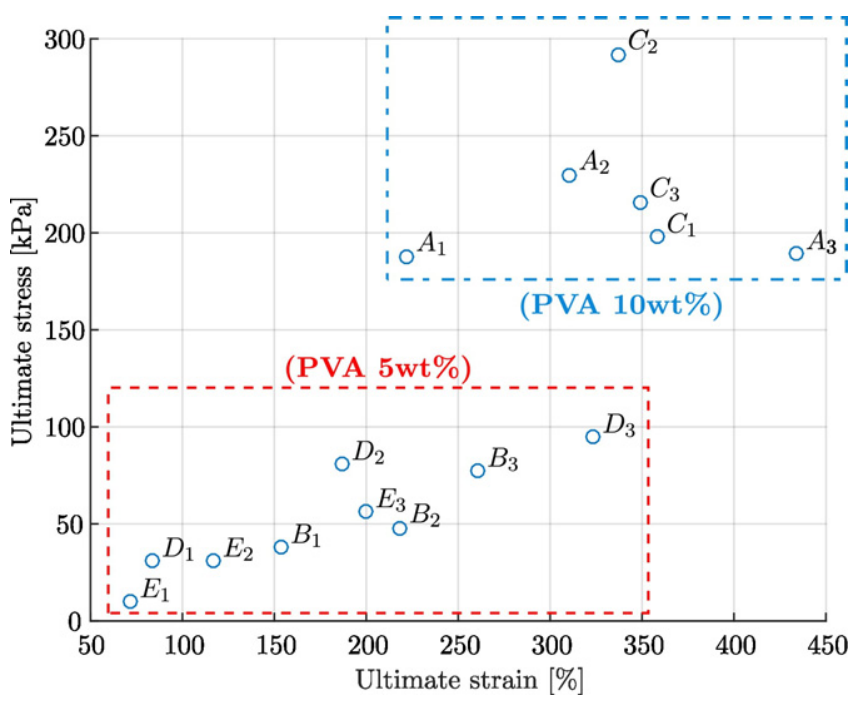

Fig. 6. Repartition of hydrogel compositions based on ultimate strain and ultimate stress.

\subsection{Modeling the mechanical response}

As presented in Section 3, the mechanical behavior of the PVA presents few viscoelastic effects. This means that it can be modeled by a pure elastic model. It is proposed to fit a hyper-elastic model on the first loading curve of the cyclic tests of the material. For this purpose, the Neo-Hookean constitutive law [20] is used to predict the experimental results previously presented. The Neo-Hookean equation is written as:

$W=C_{1}^{N H}\left(I_{1}-3\right)$

Where $W$ the strain energy, $I_{1}$ is the first invariant of the left CauchyGreen strain tensor, and $C_{1}^{N H}$ is a material parameter that can be linked to the initial modulus. $C_{1}^{N H}$ can also be considered equal to $n k T[20]$ where $n$ is the density of macromolecular chains of the amorphous phase by unit of volume $\left(\mathrm{mm}^{-3}\right), k$ the Boltzmann constant $\left(\mathrm{JK}^{-1}\right)$ and $T$ the temperature (in $\mathrm{K})$. In the case of pure shear deformation, the evolution of nominal stress is written with the stretch $\lambda$ as follows:

$\pi=2 C_{1}^{N H}\left(\lambda-\frac{1}{\lambda^{3}}\right)$

The material parameter $C_{1}^{N H}$ was fitted on the first load of the cyclic tests issued on all hydrogel compositions. As an example, Fig. 7 shows the Neo-Hookean fit corresponding to the cyclic tests performed on material $A_{1}$. The model fits the data perfectly.

Tables 4 and 5 show the mean values of material parameter $C_{1}^{\mathrm{NH}}$ for all the proposed compositions of the hydrogel in the $[1 ; 1.25]$ stretch range. 10 samples of each configuration were tested. The initial moduli are also shown, as they can be deduced from the nominal stress $\pi$ : during infinitesimal deformations, one can assume that the deformed and initial configurations coincide. The Cauchy stress $\sigma$, in pure shear test, can consequently be written as:

$\sigma=\frac{\pi}{\lambda}$

In the small deformation framework, the Cauchy stress is written:

$\sigma=\frac{E}{\varepsilon}$

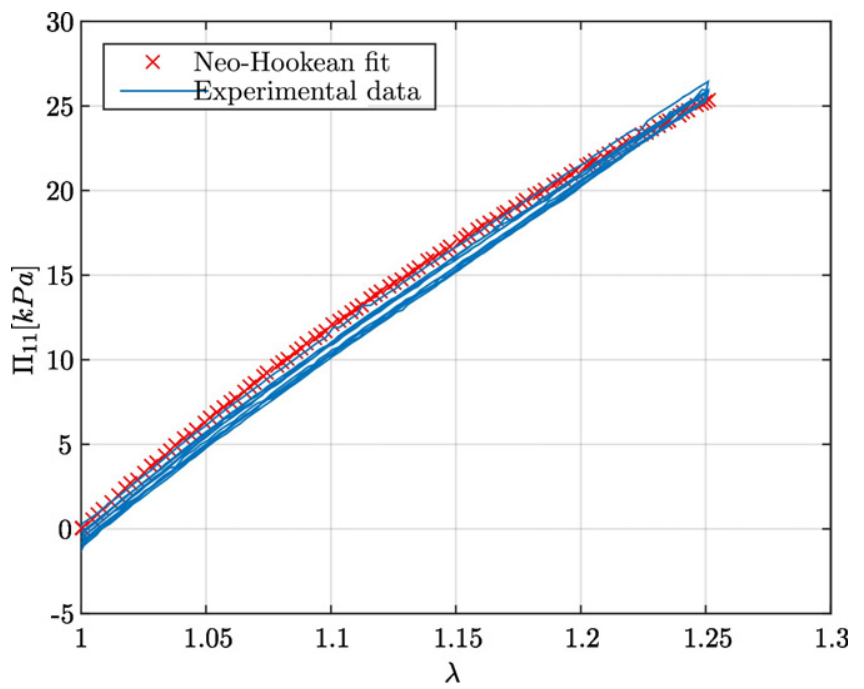

Fig. 7. Comparison of the cyclic behavior of material $A_{1}$ with the corresponding NeoHookean fit.

where $\varepsilon$ is the engineering strain measured by the testing device and $E$ the initial modulus.

Since $\lambda=1+\varepsilon$, by combining Eqs. (4) and (5), $\sigma$ can be written as:

$\left.\sigma=2 C_{1}^{N H}\left(\lambda^{2}-\frac{1}{\lambda^{2}}\right)=2 C_{1}^{N H}(1+\varepsilon)^{2}-\frac{1}{(1+\varepsilon)^{2}}\right)=8 C_{1}^{N H} \varepsilon+o(\varepsilon)$

Using Eqs. (5) and (7), the link between the $C_{1}^{\mathrm{NH}}$ material parameter and the initial modulus $E=8 C_{1}^{\mathrm{NH}}=8 \mathrm{nkT}$ is deduced. The calculated values of $n$ for a temperature of $37^{\circ} \mathrm{C}$ are also presented in Tables 4 and 5 .

The evolution of the mean values of $C_{1}^{\mathrm{NH}}$ and their standard deviation for a given PVA concentration function of the DMSO: $\mathrm{H}_{2} \mathrm{O}$ ratio is represented in Figs. 8a and 8\}b for all the freeze/thaw cycles. For the sake of comparison, materials having undergone a single freeze/thaw cycle are chosen as references. Normalizing the values obtained for 2 and 3 cycles in relation to 1 cycle allows for the evaluation of the influence of the number of freeze/thaw cycles. Fig. $8 \mathrm{c}$ and $\mathrm{d}$ show the normalized data of each material configuration.

For hydrogels containing $10 \mathrm{wt} \%$ PVA, Fig. 8a and c show an increase of $C_{1}^{N H}$ with DMSO concentration for a single freeze/thaw cycle. Also $C_{1}^{\mathrm{NH}}$ goes from being the lowest value for a DMSO concentration of $60 \%$ to being the highest for $80 \%$ DMSO. Materials having undergone more than 1 cycle present a decrease in $C_{1}^{N H}$ with the increase of DMSO.

Fig. 8b, shows that $C_{1}^{\mathrm{NH}}$ is maximal for a DMSO concentration of $60 \%$ and almost twice larger than the values calculated for DMSO concentrations of $40 \%$ and $80 \%$, which are almost equal. Also a general increase of $C_{1}^{\mathrm{NH}}$ is observed with the increased number of freeze/thaw cycles. This is more obvious in Fig. 8d: for DMSO concentrations of $40 \%$ and $80 \%, C_{1}^{\mathrm{NH}}$ increases with the number of cycles, while for the gel containing $60 \%$ DMSO, the structure seems to have stabilized after 2 freeze/thaw cycles.

For hydrogels containing 10 wt\% PVA, Fig. 8a and c show an increase of $C_{1}^{N H}$ with DMSO concentration for a single freeze/thaw cycle. Materials having undergone more than 1 cycle present a decrease in

Table 4

Values of the $\mathrm{C}_{1}^{\mathrm{NH}}$ material parameters, the initial moduli $\mathrm{E}$ and the densities of macromolecular chains by unit of volume $\mathrm{n}$ of hydrogels containing a PVA concentration of $10 \%$

\begin{tabular}{lllllll}
\hline Sample ID & $A_{1}$ & $A_{2}$ & $A_{3}$ & $C_{1}$ & $C_{2}$ & $C_{3}$ \\
\hline$C_{1}^{\mathrm{NH}}[\mathrm{kPa}]$ & 16.92 & 13.86 & 5.03 & 13.74 & 21.23 & 19.47 \\
Initial modulus $[\mathrm{kPa}]$ & 135.39 & 110.92 & 40.29 & 109.92 & 169.84 & 155.75 \\
$n\left[10^{14} \mathrm{~mm}^{-3}\right]$ & 39.53 & 32.38 & 11.75 & 32.10 & 49.60 & 45.49
\end{tabular}


Table 5

Values of the $C_{1}^{\mathrm{NH}}$ material parameters, the initial moduli E and the densities of macromolecular chains by unit of volume n of hydrogels containing a PVA concentration of $5 \%$.

\begin{tabular}{|c|c|c|c|c|c|c|c|c|c|}
\hline Sample ID & $E_{1}$ & $E_{2}$ & $E_{3}$ & $D_{1}$ & $D_{2}$ & $D_{3}$ & $E_{1}$ & $E_{2}$ & $E_{3}$ \\
\hline $\mathrm{C}_{1}^{\mathrm{NH}}[\mathrm{kPa}]$ & 2.94 & 3.35 & 4.24 & 3.06 & 7.23 & 6.93 & 2.27 & 3.43 & 3.74 \\
\hline Initial modulus [kPa] & 23.54 & 26.80 & 33.94 & 48.49 & 57.84 & 55.46 & 18.20 & 27.49 & 29.95 \\
\hline$n\left[10^{14} \mathrm{~mm}^{-3}\right]$ & 6.89 & 7.82 & 9.90 & 14.16 & 16.89 & 16.19 & 5.30 & 8.01 & 8.73 \\
\hline
\end{tabular}

$C_{1}^{N H}$ with the increase of DMSO. Also $C_{1}^{N H}$ goes from being the lowest value for a DMSO concentration of $60 \%$ to being the highest for $80 \%$ DMSO.

Fig. 8b, shows that $C_{1}^{\mathrm{NH}}$ is maximal for a DMSO concentration of $60 \%$ and almost twice larger than the values calculated for DMSO concentrations of $40 \%$ and $80 \%$, which are almost equal. Also a general increase of $\mathrm{C}_{1}^{\mathrm{NH}}$ is observed with the increased number of freeze/thaw cycles. This is more obvious in Fig. $8 \mathrm{~d}$ : for DMSO concentrations of $40 \%$ and $80 \%, C_{1}^{\mathrm{NH}}$ increases with the number of cycles, while for the gel containing $60 \%$ DMSO, the structure seems to have stabilized after 2 freeze/thaw cycles. In general, it is safe to say that the number of freeze/thaw cycles imposed on a material does have an influence, but from a mechanical point of view their effect varies with the initial composition of the material.

Tables 4 and 5 show the mean values of material parameter $C_{1}^{\mathrm{NH}}$, the deduced initial moduli and the density of macromolecular chains of the amorphous phase by unit of volume $n$ for all the proposed compositions of the hydrogel. Analysis of Table 4 shows that $C_{1}^{\mathrm{NH}}$ decreases for materials containing $10 \mathrm{wt} \%$ PVA and 80\% DMSO with the number of freeze/ thaw cycles. This translates into a decrease of the density of macromolecular chains of the amorphous phase, which might be due to crystal phase outgrowing the amorphous phase. For hydrogels containing $60 \%$ DMSO however, $C_{1}^{N H}$, as well as $n$, are maximal for 2 freeze/thaw cycles

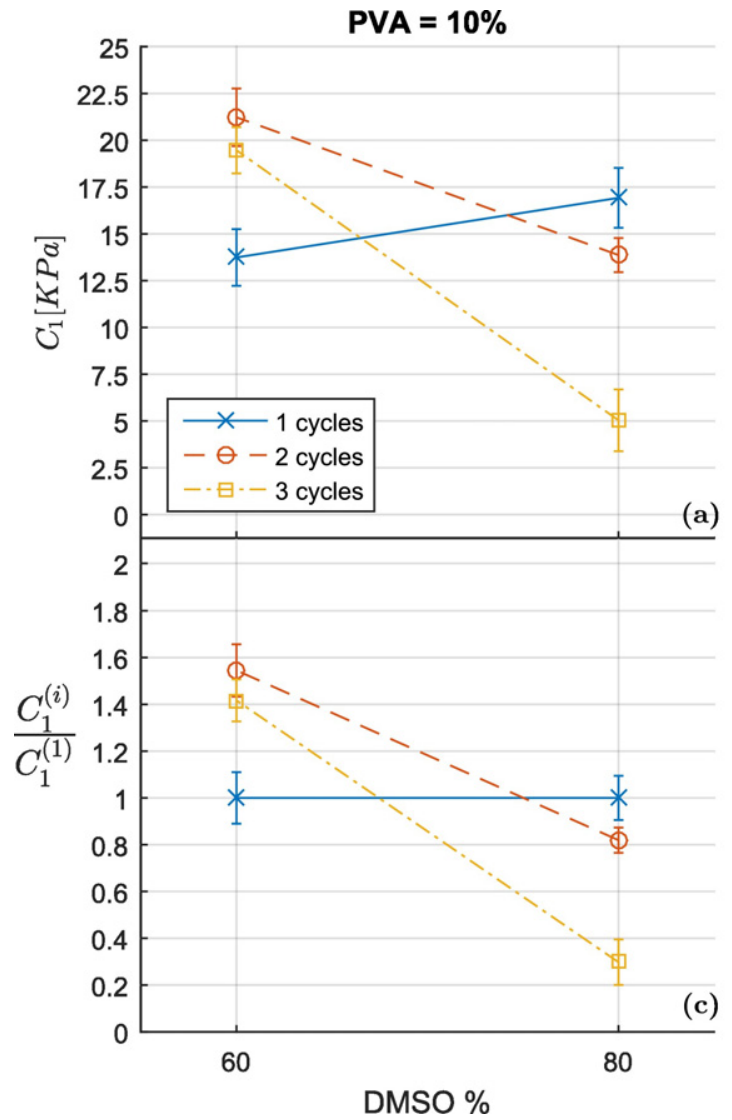

and minimal for a single one. This is due to the fact that the gelation time is smallest for $60 \%$ DMSO, which might lead to a fully accomplished amorphous phase by the second cycle and an increase of the crystal phase during the third cycle.

For materials consisting of $5 \mathrm{wt} \% \mathrm{PVA}, C_{1}^{\mathrm{NH}}$ increases with the number of freeze/thaw cycles for all the compositions except for sample $D_{2}$ which is slightly larger than $D_{3}$. It is also highest for material $D$ that contains an initial DMSO concentration of $60 \%$. $n$ behaves in a similar way thus the explanation proposed for material $C$ also applies for materials $B, D$ and $E$.

\section{Conclusion}

This study investigated the effects of the initial processing parameters of PVA/DMSO hydrogels on their mechanical behavior. A mechanical study in a controlled environment consisting of cyclic and failure tensile tests was made. A tank containing a thermostatic aqueous medium was developed specifically to account for the hydrophilic behavior of the material. This allowed for the observation of different phenomena such as hyperelasticity, softening and residual strain. The results show an influence of the number of freeze/thaw cycles applied to each material configuration but a strong dependence on the initial solution, especially for materials containing larger concentrations of PVA. In general, a

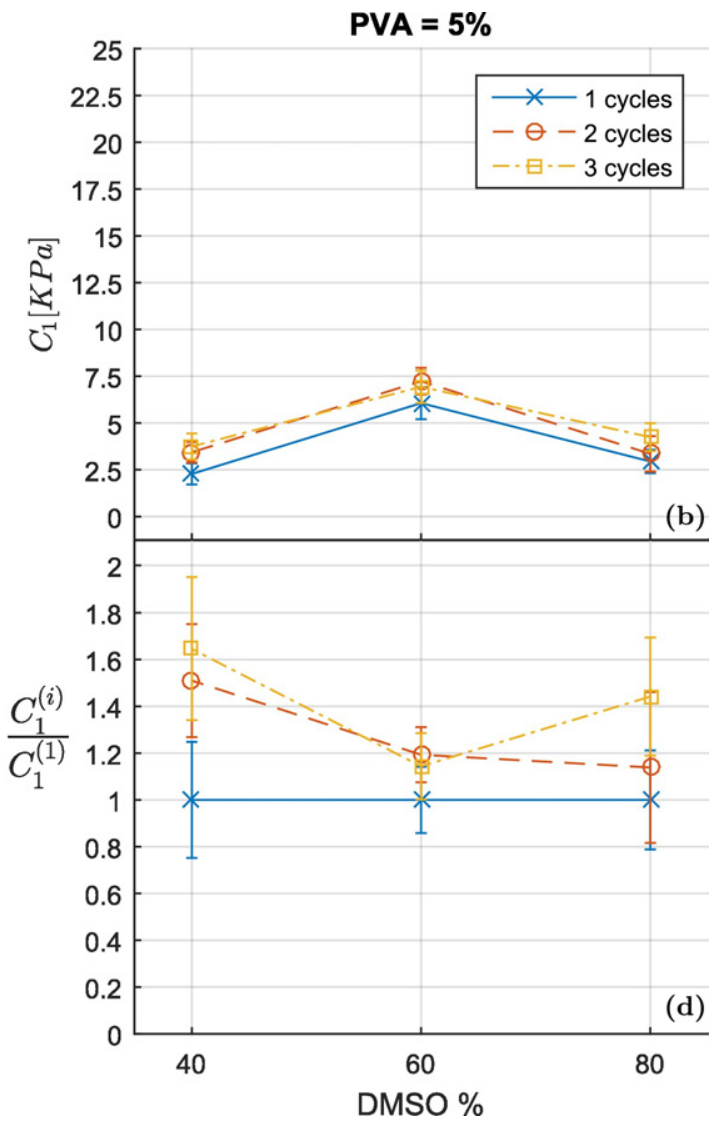

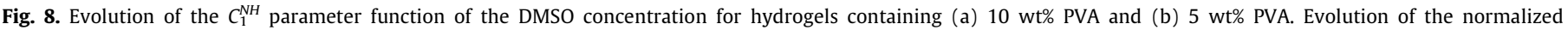

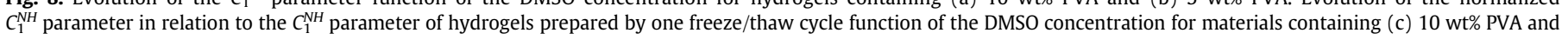
(d) $5 \mathrm{wt} \%$ PVA. 
loss of mechanical properties was observed with decreasing PVA concentrations. In the tested strain range, the hydrogels exhibited very little visco-elasticity which motivated the use of the Neo-Hookean hyperelastic model to reproduce the results. This was achieved with great success and allowed for the measurement of the elastic modulus of the different materials function of their respective elaboration parameters. The strongest hydrogel was obtained for a PVA concentration of $10 \mathrm{wt} \%$, a DMSO: $\mathrm{H}_{2} \mathrm{O}$ ratio of $60: 40$ and two freeze/thaw cycles. It is worth noting that the elastic behavior of PVA hydrogels compares well with soft tissue, thus making them excellent candidates for producing phantoms with controlled mechanical behaviors. In perspective, a link between the mechanical properties of PVA hydrogels and their crystallinity would allow for a general description of their behavior, independently of the processing parameters.

\section{Acknowledgments}

The authors would like to thank Dr. Jacques Thelu who, through enlightening discussions, provided insight and expertise that assisted this research.

\section{References}

[1] V.G. Kadajji, G.V. Betageri, Water soluble polymers for pharmaceutical applications, Polymer 3 (4) (1972-2009) 2011.

[2] S. Jiang, Z. Su, X. Wang, S. Liu, Y. Yu, Development of a new tissue-equivalent material applied to optimizing surgical accuracy, Mater. Sci. Eng. C 33 (7) (2013) 3768-3774.

[3] C.M. Hassan, N.A. Peppas, Structure and Applications of Poly(vinyl alcohol) Hydrogels Produced by Conventional Crosslinking or by Freezing/Thawing Methods, 2000153.

[4] N.A. Peppas, N.K. Mongia, Ultrapure poly(vinyl alcohol) hydrogels with mucoadhesive drug delivery characteristics, Eur. J. Pharm. Biopharm. 43 (1) (1997) 51-58.
[5] S.H. Hyon, W.I. Cha, Y. Ikada, M. Kita, Y. Ogura, Y. Honda, Poly(vinyl alcohol) hydrogels as soft contact lens material, J. Biomater. Sci. Polym. Ed. 5 (5) (1994) 397-406.

[6] M. Kobayashi, J. Toguchida, M. Oka, Development of the shields for tendon injury repair using poly(vinyl alcohol) - hydrogel (PVA-H), J. Biomed. Mater. Res. 58 (4) (2001) 344-351.

[7] J.S. Bach, F. Detrez, M. Cherkaoui, S. Cantournet, D.N. Ku, L. Corté, Hydrogel fibers for ACL prosthesis: design and mechanical evaluation of PVA and PVA/UHMWPE fiber constructs, J. Biomech. 46 (8) (2013) 1463-1470.

[8] Z.Q. Gu, J.M. Xiao, X.H. Zhang, The development of artificial articular cartilage PVAhydrogel, Biomed. Mater. Eng. 8 (2) (1998) 75-81.

[9] N.A. Peppas, Hydrogels in Medicine and Pharmacy, Vol. l, CRC Press Inc., Boca Raton, Florida, 1986 (1987 (Vols. 2 and 3). Volume 21. John Wiley \& Sons, Ltd., 1989).

[10] A. Danno, Gel formation of aqueous solution of poly(vinyl alcohol) irradiated by gamma rays from cobalt-60, J. Phys. Soc. Jpn. 13 (7) (1958) 722-727.

[11] S.R. Stauffer, N.A. Peppas, Poly(vinyl alcohol) hydrogels prepared by freezingthawing cyclic processing, Polymer 33 (18) (Sep 1992) 3932-3936.

[12] H. Trieu, S. Qutubuddin, Poly(vinyl alcohol) hydrogels: 2. Effects of processing parameters on structure and properties, Polymer 36 (13) (Jan 1995) 2531-2539.

[13] S.H. Hyon, W.I. Cha, Y. Ikada, Preparation of transparent poly(vinyl alcohol) hydrogel, Polym. Bull. 22 (2) (1989) 119-122.

[14] T. Tanigami, K. Murase, K. Yamaura, et al., Polymer 35 (12) (1994) 2573-2578.

[15] L. Mandelkern, The effect of molecular weight on the crystallization, melting, and morphology of long-chain molecules, Journal of Polymer Science Part C: Polymer Symposia 15 (1967) 129-162.

[16] D.J. Charlton, J. Yang, K.K. Teh, A Review of Methods to Characterize Rubber Elastic Behavior for Use in Finite Element Analysis, 2012.

[17] J. Diani, B. Fayolle, P. Gilormini, A review on the Mullins effect, Eur. Polym. J. 45 (3) (2009) 601-612.

[18] F. Yokoyama, I. Masada, K. Shimamura, T. Ikawa, K. Monobe, Morphology and structure of highly elastic poly(vinyl alcohol) hydrogel prepared by repeated freezingand-melting, Colloid Polym. Sci. 264 (7) (1986) 595-601.

[19] H. Hoshino, S. Okada, H. Urakawa, K. Kajiwara, Gelation of poly(vinyl alcohol) in dimethyl sulfoxide/water solvent, Polym. Bull. 37 (2) (Aug 1996) 237-244.

[20] L.R.J. Treloar, The elasticity of a network of long-chain molecules I and II, Trans. Faraday Soc. 36 (1942) 36-41. 